\title{
Comprehensive analysis of differentially expressed long non-coding RNAs in non-small cell lung cancer
}

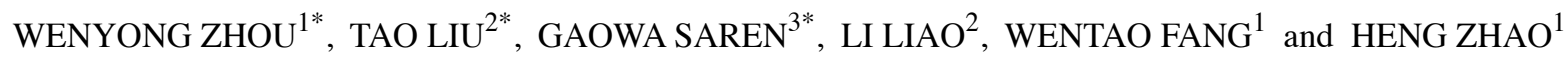 \\ ${ }^{1}$ Department of Thoracic Surgery, Shanghai Chest Hospital, Shanghai Jiao Tong University, Shanghai 200030; \\ Departments of ${ }^{2}$ Oncology and ${ }^{3}$ Intensive Care, Huashan Hospital, Fudan University, Shanghai 200040, P.R. China
}

Received September 7, 2018; Accepted April 17, 2019

DOI: $10.3892 / 01.2019 .10414$

\begin{abstract}
Non-small cell lung cancer (NSCLC) is the primary subtype of lung cancer. Long non-coding RNAs (lncRNAs) have been reported to serve prominent roles in cancer progression. However, the expression patterns and potential roles of IncRNAs in NSCLC remain to be elucidated. In the present study, four public datasets were analyzed to identify differentially expressed lncRNAs (DElncs) in NSCLC. A further dataset, GSE19188, was analyzed to validate the findings. A total of 38 upregulated and 31 downregulated lncRNAs were identified in NSCLC, compared with samples from healthy controls. Among these, 12 lncRNAs were associated with the progression of NSCLC, and dysregulated between high grade (stage III and IV) and low grade (stage II) NSCLC samples. Moreover, dysregulation of lncRNA-SIGLEC17P, GGTA1P, A2M-AS1, LINC00938, GVINP1, LINC00667 and TMPO-AS1 was associated with overall survival time in patients with NSCLC. Co-expression analyses, combined with the construction of protein-protein interaction networks, were performed to reveal the potential roles of key lncRNAs in NSCLC. The present study revealed a series of IncRNAs involved in the progression of NSCLS, which may serve as novel biomarkers for the disease.
\end{abstract}

\section{Introduction}

With the development of high-throughput methods, a number of studies have revealed the roles of non-coding RNAs, including microRNAs (miRNA) (1), long non-coding RNAs (lncRNAs) (2) and small nucleolar RNAs (snoRNAs) (3).

Correspondence to: Dr Wentao Fang or Dr Heng Zhao, Department of Thoracic Surgery, Shanghai Chest Hospital, Shanghai Jiao Tong University, 241 West Huaihai Road, Shanghai 200030, P.R China

E-mail: vv_fangwentao@163.com

E-mail: dr_zhaoheng@yeah.net

*Contributed equally

Key words: long non-coding RNA, protein-protein interaction network, non-small cell lung cancer, expression profiling, biomarker
lncRNAs are a subclass of RNA transcripts $>200$ bps in length (4). Emerging studies have indicated that lncRNAs are abnormally expressed in various types of human cancer, including prostate cancer (5), breast cancer (6), hepatocellular carcinoma (7) and lung cancer (8). These lncRNAs were involved in multiple biological processes, including the cell cycle, invasion, proliferation and drug resistance, by influencing the targets of transcriptional (cis/trans) and post-transcriptional regulation (9). Specific lncRNAs, including GHET1 (10), LINP1 (11), NEAT1 (12) and MIR100HG (13) were reported to be oncogenes. However, various other lncRNAs, including GAS5 (14) and LINC00961 (15), were implicated in cancer suppression. Therefore, exploring the functions of lncRNAs may provide novel insights to identify diagnostic and prognostic markers of cancer.

Lung cancer has become the leading cause of cancer-associated mortality worldwide (16), of which non-small cell lung cancer (NSCLC) is the primary subtype (16). Despite the development of various treatment methods, including radiotherapy, chemotherapy and targeted therapy, the five-year survival rate of NSCLC remains low, at $\sim 15 \%$ (17). In previous reports, IncRNAs were identified as novel regulators of lung cancer progression; the transforming growth factor $\beta$-induced lncRNA-TBILA promoted NSCLC progression via cis-regulation of human germinal center-associated lymphoma HGAL, and activation of S100A7/JAB1 (18). However, in NSCLC, the potential roles of a great number of lncRNAs remain unknown.

The aim of the present study was to identify novel therapeutic and prognostic biomarkers for NSCLC. Previously published datasets were analyzed to identify differentially expressed lncRNAs (DElnc). To determine the functions of these DElncs, a series of bioinformatics analyses were performed, including Gene Ontology (GO) and Kyoto Encyclopedia of Genes and Genomes analysis.

\section{Materials and methods}

lncRNA classification pipeline. A pipeline described by Zhang et al (19) was employed to re-annotate the microarray data. Entries labeled as 'NR' or annotated with 'IncRNA', 'processed transcripts', 'non-coding' or 'misc_RNA' in Ensembl annotations, were retained. 
Microarray data and pre-processing. The following datasets were downloaded from the Gene Expression Omnibus (GEO) database (https://www.ncbi.nlm.nih.gov/geo/) to identify DElncs: GSE19804, GSE27262, GSE18842 and GSE19188. GSE19804 was reported by Lu et al (20) and contained 65 normal samples and 91 female lung cancer samples; GSE27262 included 25 normal and 25 stage I lung adenocarcinoma patients (21); GSE18842 included 45 normal and 46 NSCLC samples (22). A further dataset, GSE19188, which includes 65 normal and 91 lung cancer samples, was used for validation of the findings (23). In addition, The Cancer Genome Atlas Lung Adenocarcinoma dataset (TCGA-LUAD), based on RNA-sequence methodology was also downloaded to analyze the expression patterns of lncRNAs in LUAD samples. In the present study, the lncRNAs whose expression in NSCLC samples were higher than that in normal samples (with a fold change $(\mathrm{FC}) \geq 2$ and $\mathrm{P}<0.05$ ), were considered to be upregulated. The lncRNAs whose expression in NSCLC samples was lower than that in normal samples (with an $\mathrm{FC} \leq 0.5$ and $\mathrm{P}<0.05$ ), were considered to be downregulated.

Functional group analysis. Bioinformatics analysis was conducted using the Database for Annotation, Visualization and Integrated Discovery system (DAVID version 6.8; http://david. ncifcrf.gov/), to identify the relevant biological functions of any high-throughput gene functional analysis (24). $\mathrm{P}<0.05$ was considered to indicate a statistically significant difference.

Protein-protein interaction (PPI) network and module analysis. Search Tool for the Retrieval of Interacting Genes/Proteins (STRING) online software was used to construct PPI networks (https://string-db.org/cgi/input. pl?sessionId=AUH42ZEZwajP\&input_page_show_search=on). PPIs with a combined score $>0.4$ were considered to be significant. Cytoscape software (https://cytoscape.org/) was used to visualize the PPI networks.

Survival analysis. The Kaplan-Meier plotter (http://www. kmplot.com/analysis) is a public dataset including 54,675 genes on survival using 2,437 lung cancer samples, with a mean follow-up period of 49 months. The median expression of lncRNAs was selected as the cut-off point to divide patients with NSCLC into high- and low-expression groups.

Statistical analysis. Data are presented as the mean \pm standard deviation. All statistical analyses were performed using SPSS 17.0 software (SPSS, Inc.). Statistical comparisons between groups were performed using the Mann-Whitney $\mathrm{U}$ test. $\mathrm{P}<0.05$ was considered to indicate a statistically significant difference.

\section{Results}

Identification of DElncs in NSCLC. In order to identify key lncRNAs involved in the progression of NSCLC, comprehensive analysis of four public datasets were performed. A total of 638 upregulated and 294 downregulated lncRNAs were identified in the GSE19804 dataset (Fig. 1A); 525 upregulated and 216 downregulated lncRNAs were identified in the GSE27262 dataset (Fig. 1B); 379 upregulated and 508 downregulated
IncRNAs were identified in the GSE18842 dataset (Fig. 1C); and 489 upregulated and 223 downregulated lncRNAs were identified in TCGA dataset (Fig. 1D). Hierarchical clustering revealed systematic variations in the expression of lncRNAs in NSCLC samples.

Integrated analysis of DElncs in NSCLC was also performed. The upregulation of 38 lncRNAs (Fig. 2A), and the downregulation of 31 lncRNAs (Fig. 2B) was observed in lung cancer compared with normal, healthy samples. Of note, a number of lncRNAs identified as prominent regulators in lung cancer progression were upregulated, including TUG1, DLEU2 and DANCR. However, the functions of the majority of lncRNAs in human disease require further investigation.

Verification of DElncs in NSCLC. In order to verify the expression pattern of the aforementioned DElncs in NSCLC, the validation dataset GSE19188 was analyzed. Upregulation of 38, and downregulation of 31 lncRNAs in the training datasets were identified as dysregulated in GSE19188. Hierarchical clustering revealed systematic variations in the expression of lncRNAs in the NSCLC, by analysis of GSE19188 (Fig. 2C).

Identification of IncRNAs associated with the progression of NSCLC. Associations between the expression of 69 DElncs and the clinicopathological features of NSCLC were determined. The expression levels of lncRNA-GVINP1, RPL32P3, EPB41L4A-AS2, ILF3-AS1, LINC00938, LINC00667, A2M-AS1, GUSBP11, GGTA1P, BCRP3 and SIGLEC17P were suppressed, while lncRNA-TMPO-AS1 expression was upregulated in high grade (stage III and IV) in comparison to low grade (stage II) NSCLC samples (Fig. 3).

Dysregulation of DElncs is associated with altered survival time of patients with NSCLC. To evaluate the prognostic value of DElncs in NSCLC, Kaplan-Meier analysis was performed using TCGA LUAD dataset. Patients with NSCLC were divided into low- and high-lncRNA expression groups, with the median expression level selected as the cut-off. Among these lncRNAs, higher expression of lncRNA-SIGLEC17P, GGTA1P, A2M-AS1, ILF3-AS1, LINC00938 and GVINP1, in addition to lower expression of IncRNA-TMPO-AS1, was associated with longer survival time in patients with LUAD (Fig. 4). Furthermore, the Kaplan-Meier-plotter database was used to ascertain the potential prognostic values of the aforementioned lncRNAs. It was revealed that higher expression levels of IncRNA-SIGLEC17P, GGTA1P, A2M-AS1, LINC00667 and GVINP1, and lower expression levels of lncRNA-ILF3-AS1, LINC00938 and TMPO-AS1 were associated with higher survival rates in patients with NSCLC (Fig. 5). Collectively, these results suggested that DElncs may serve as novel biomarkers for the prognosis of patients with NSCLC.

Construction of key lncRNA-associated PPI networks in NSCLC. Co-expression analysis was performed for the construction of PPI networks to reveal the potential roles and molecular mechanisms of key lncRNAs in NSCLC. The Pearson's correlation coefficient was calculated for the lncRNA-mRNAs, using their expression levels in TCGA dataset. IncRNA-mRNAs pairs with $|R|>0.7$ were considered 

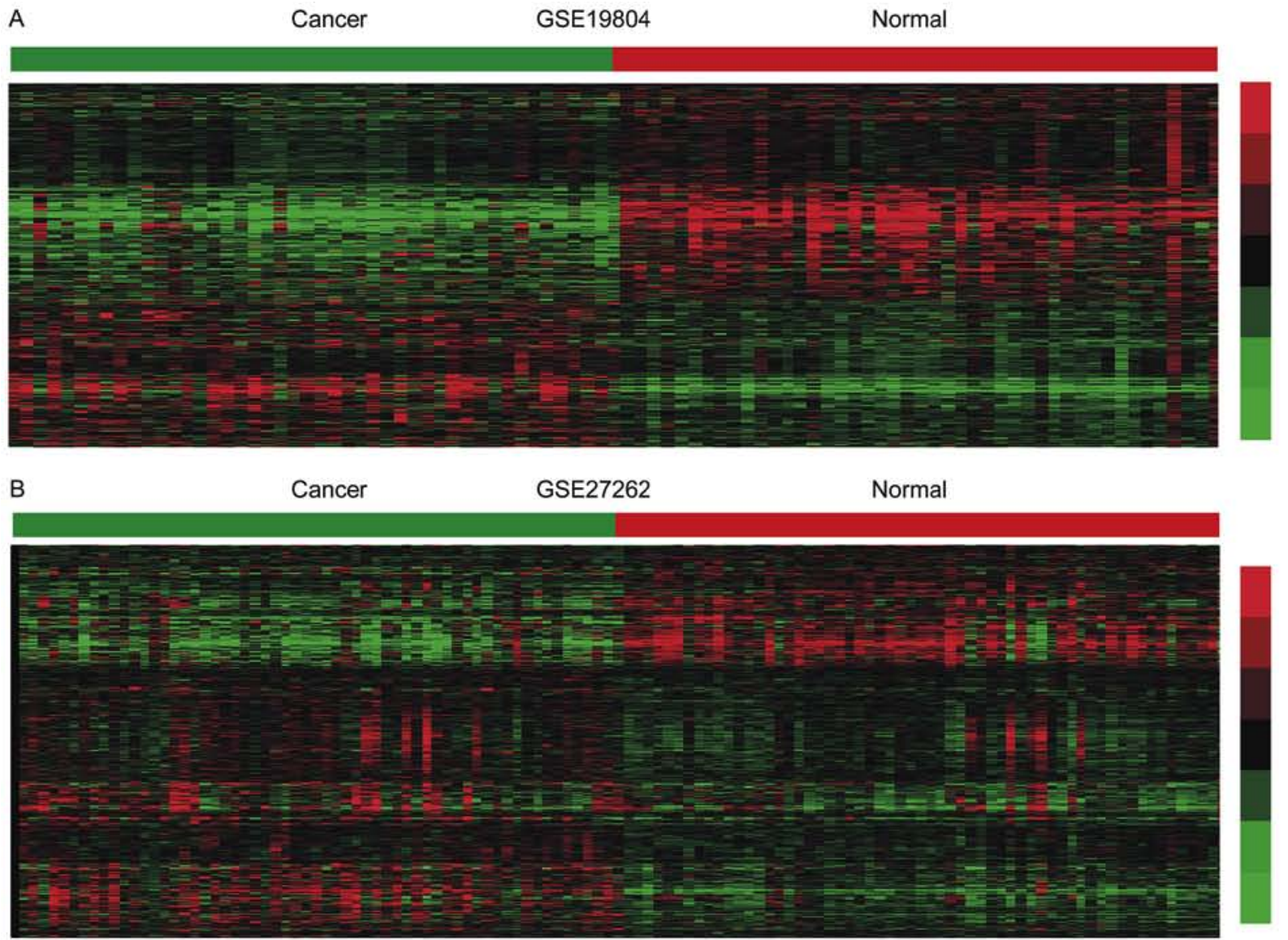

\section{C}
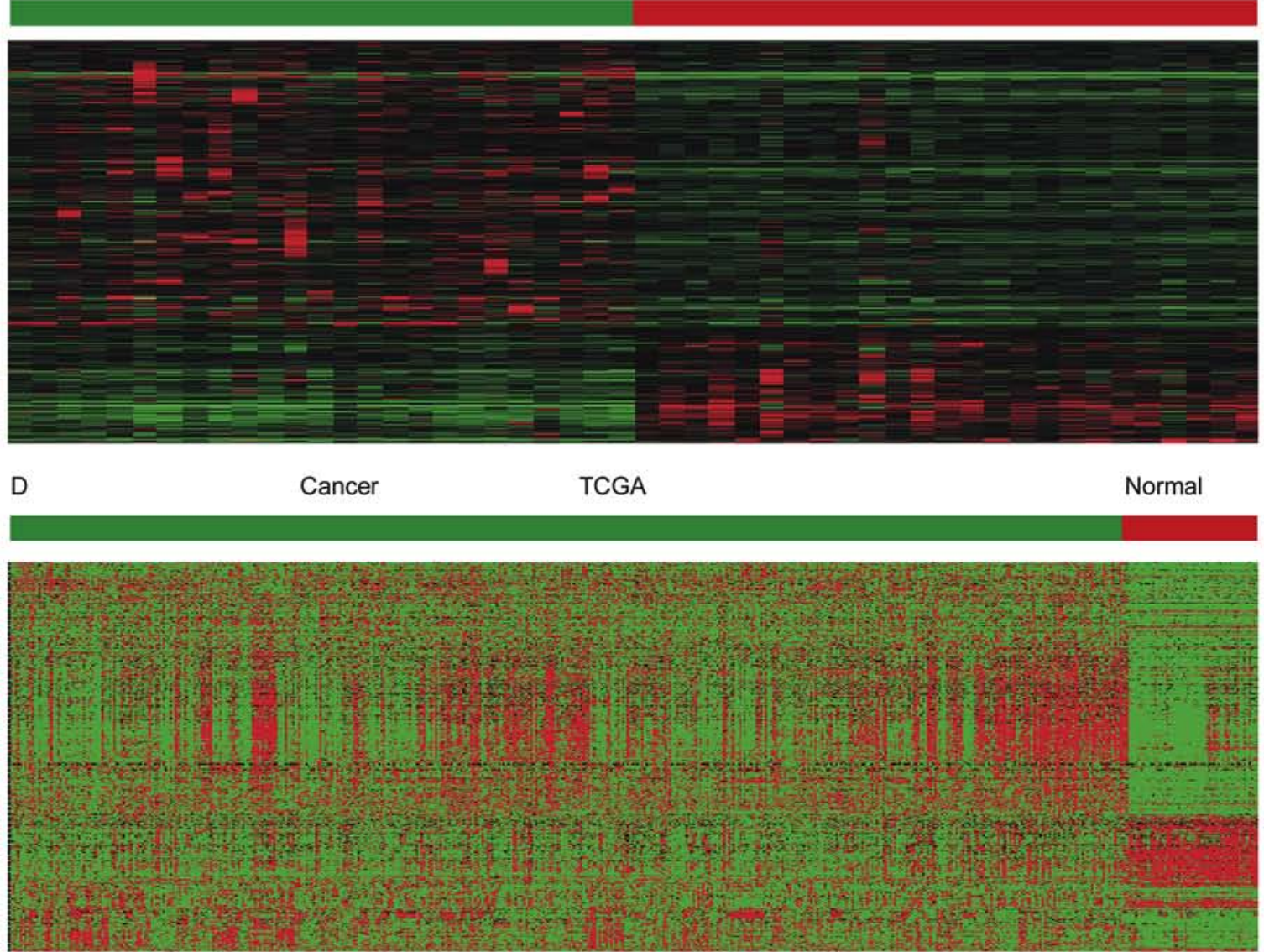

Figure 1. Identification of differentially expressed long non-coding RNAs in NSCLC. Differentially expressed lncRNAs in NSCLC vs. normal tissues, obtained from (A) GSE19804, (B) GSE27262, (C) GSE18842 and (D) TCGA LUAD datasets are represented with heatmaps. Red indicates high relative expression and green indicates low relative expression. NSCLC, non-small cell lung cancer; TCGA LUAD, The Cancer Genome Atlas Lung Adenocarcinoma. 

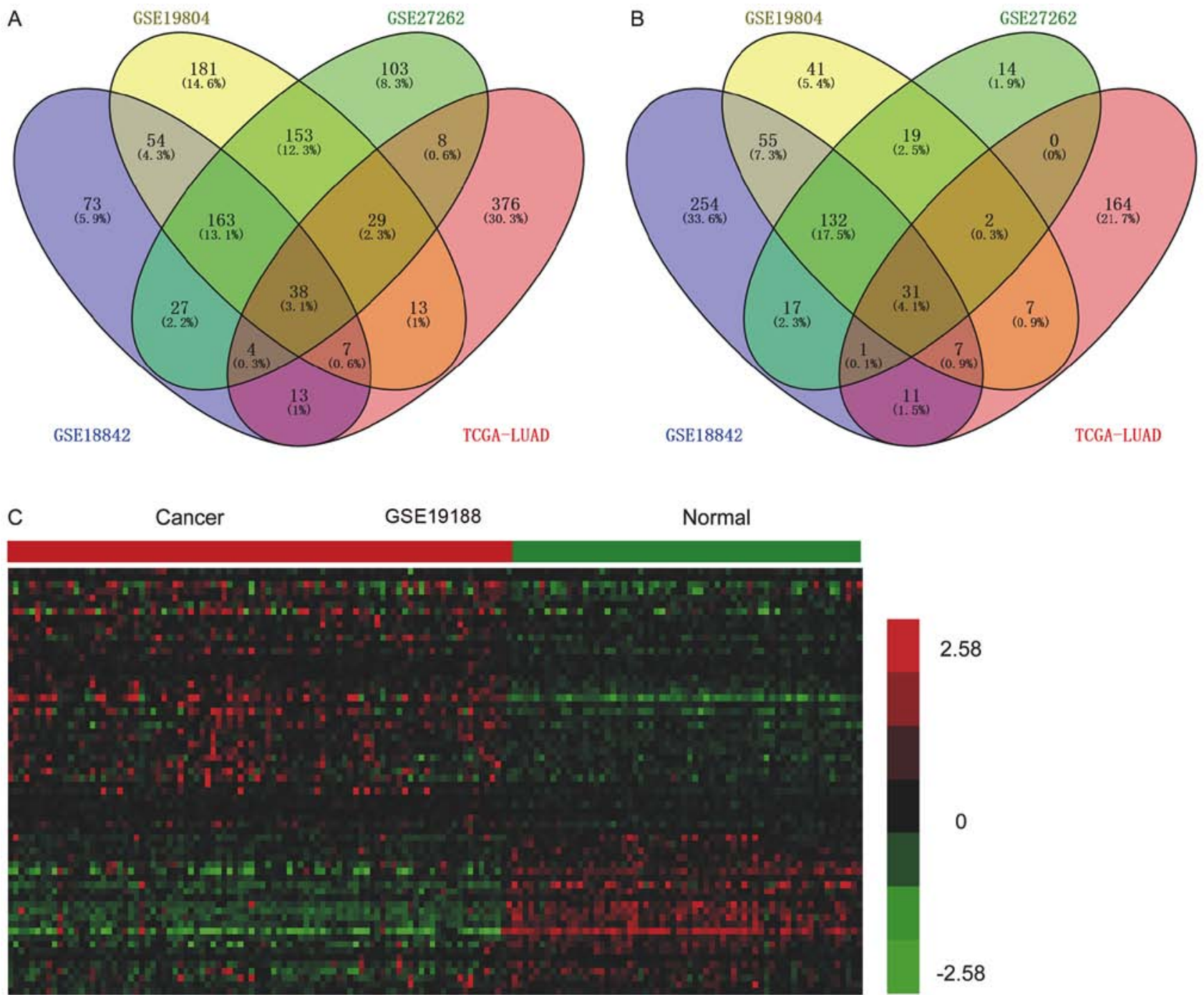

Figure 2. Verification of DElncs associated with NSCLC, using a validation dataset. Four-way Venn diagrams display the overlap between (A) upregulated and (B) downregulated lncRNAs associated with NSCLC, from the analysis of GSE18842, GSE19804, GSE27262 and TCGA LUAD. (C) Verification of the DElncs in NSCLC using GSE19188. Red indicates high relative expression and green indicates low relative expression. DElncs, differentially expressed lncRNAs; NSCLC, non-small cell lung cancer; TCGA LUAD, The Cancer Genome Atlas Lung Adenocarcinoma.

to be reliable. Subsequently, the STRING database was used to investigate PPIs among key lncRNA genes that were co-expressed (combined score $>0.4$ ).

The A2M-AS1 mediated PPI network included 67 proteins and 282 edges (Fig. 6A). The GVINP1 mediated PPI network included 28 proteins and 378 edges (Fig. 6B). The LINC00667 mediated PPI network included 23 proteins and 244 edges (Fig. 6C). The GGTA1P mediated PPI network included 47 proteins and 329 edges (Fig. 6D). TMPO-AS1mediated PPI network included 118 proteins and 6075 edges (Fig. 6E). The ILF3-AS1 mediated PPI network included 13 proteins and 78 edges (Fig. 6F). The SIGLEC17P mediated PPI network included 65 proteins and 560 edges (Fig. 7A). The LINC00938 mediated PPI network included 33 proteins and 176 edges (Fig. 7B).

Functional analysis of key lncRNAs in NSCLC. Bioinformatics analysis was performed using the DAVID system. GO analysis revealed that IncRNA-GVINP1 was associated with the regulation of B cell receptor signaling pathway, inflammatory response, positive regulation of $\mathrm{T}$ cell proliferation, immune response and chemotaxis (Fig. 8A); A2M-AS1 was associated with cell adhesion, angiogenesis, positive regulation of GTPase activity, leukocyte migration and vasculogenesis (Fig. 8B); SIGLEC17P was associated with cell adhesion, leukocyte migration, signal transduction, positive regulation of GTPase activity and angiogenesis (Fig. 8C); ILF3-AS1 was involved in regulating cilium morphogenesis, cilium assembly, cilium movement, axoneme assembly and protein homotetramerization (Fig. 8D); TMPO-AS1 was associated with cell division, DNA replication, mitotic nuclear division, sister chromatid cohesion and DNA repair (Fig. 8E); LINC00938 was involved in regulating transcription, cilium morphogenesis, intraciliary retrograde transport, skin development and cilium assembly (Fig. 8F); LINC00667 was involved in regulating transcription, apoptotic process, one-carbon metabolic process, negative regulation of endothelial cell migration and mitotic cytokinesis (Fig. 8G); and GGTA1P was associated with leukocyte migration, inflammatory response, cell adhesion, immune response and signal transduction (Fig. $8 \mathrm{H}$ ). 

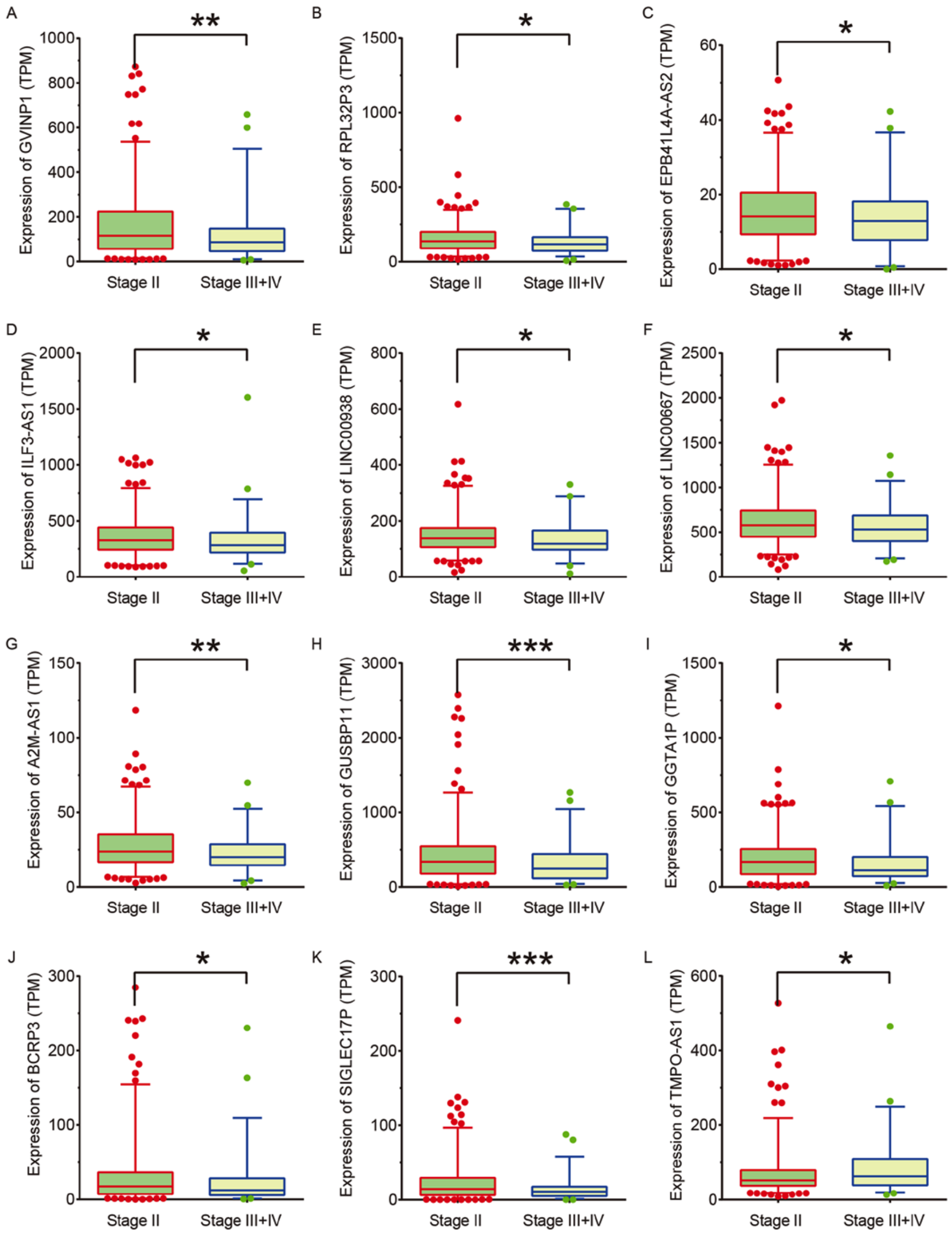

Figure 3. Identification of 1ncRNAs associated with NSCLC progression. (A) lncRNA-GVINP1, (B) RPL32P3, (C) EPB41L4A-AS2, (D) ILF3-AS1, (E) LINC00938, (F) LINC00667, (G) A2M-AS1, (H) GUSBP11, (I) GGTA1P, (J) BCRP3, and (K) SIGLEC17P expression were suppressed, and (L) TMPO-AS1 expression was upregulated in high grade (stage III and stage IV) compared with low grade (stage II) NSCLC samples. "P $<0.05 ;{ }^{* *} \mathrm{P}<0.01$; ${ }_{* * * *} \mathrm{P}<0.001$. DElncs, differentially expressed lncRNAs; NSCLC, non-small cell lung cancer; TPM, transcripts per kilobase (of exon model per million mapped reads). 

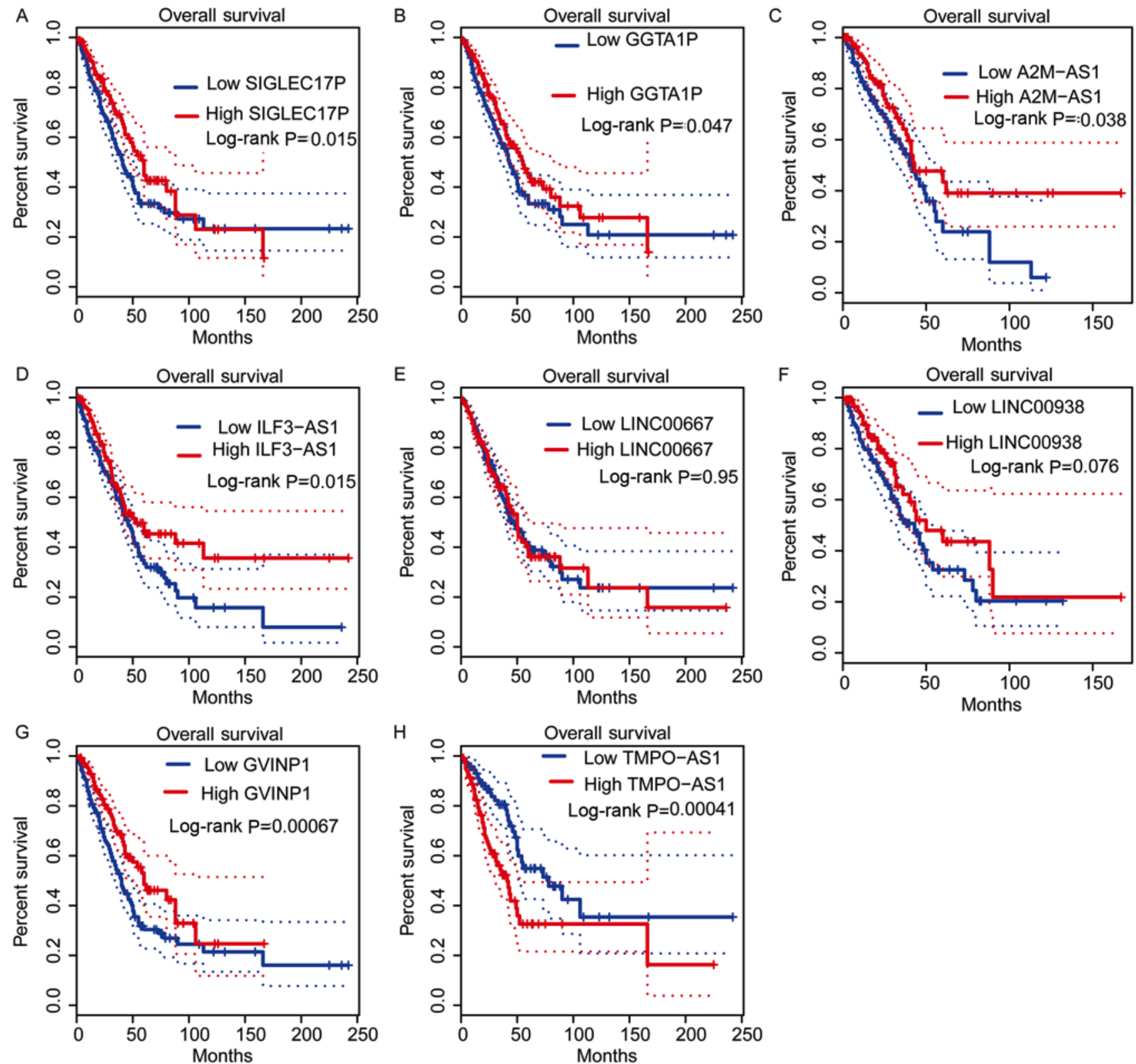

Figure 4. Dysregulation of DElncs is associated with survival time in NSCLC, as determined by analysis of TCGA dataset. TCGA LUAD analysis revealed that higher expression levels of (A) lncRNA-SIGLEC17P, (B) GGTA1P, (C) A2M-AS1, (D) ILF3-AS1 were associated with longer survival times in patients with LUAD. However, the expression of (E) lncRNA-LINC00667 was not significantly associated with prognosis in LUAD patients. Higher expression levels of (F) LINC00938 and (G) GVINP1, and lower expression of (H) TMPO-AS1 were associated with longer survival times in patients with LUAD. Red dotted lines represent the $95 \%$ confidence interval of the high group survival curves. Blue dotted lines represent the $95 \%$ confidence interval of the low group survival curves. Differentially expressed lncRNAs; NSCLC, non-small cell lung cancer; TCGA LUAD, The Cancer Genome Atlas Lung Adenocarcinoma; lncRNA, long non-coding RNA.

\section{Discussion}

NSCLC is one of the leading causes of cancer-associated mortality worldwide. Previous studies have demonstrated that lncRNAs serve a prominent role in the progression of NSCLC, as either oncogenes or tumor suppressors; IncRNA-MALAT-1 enhanced NSCLC cell motility (25) and lncRNA-CCAT2 promoted the invasion of NSCLC cells (26). However, IncRNA-SIK1-LNC suppressed the proliferative and invasive abilities of NSCLC cells (27). IncRNA-PICART1 suppressed the proliferation of NSCLC cells by inhibiting JAK2/signal transducer and activator of transcription 3 signaling (28). Of note, other reports have demonstrated that the dysregulation of specific lncRNAs may serve as an indicator of NSCLC. For instance, IncRNA-HOTAIR (29), MALAT1 (25), CCAT2 (26) and H19 (30) were upregulated, and lncRNA-MEG3 (31) and TUG1 (32) were downregulated in lung cancer. However in NSCLC, the expression patterns and molecular functions of the majority of IncRNAs remained to be investigated.

In the present study, using various public datasets, DElncs were identified as diagnostic biomarkers for NSCLC. The upregulation of 38 lncRNAs was observed in NSCLC compared with normal tissues, in addition to the suppression of a further 31 lncRNAs. Among these lncRNAs, HCG11 (33), CASC15 (34), TUG1 (32), DLEU2 (35) and DANCR (36) were dysregulated during cancer progression. IncRNA-HCG11 was 

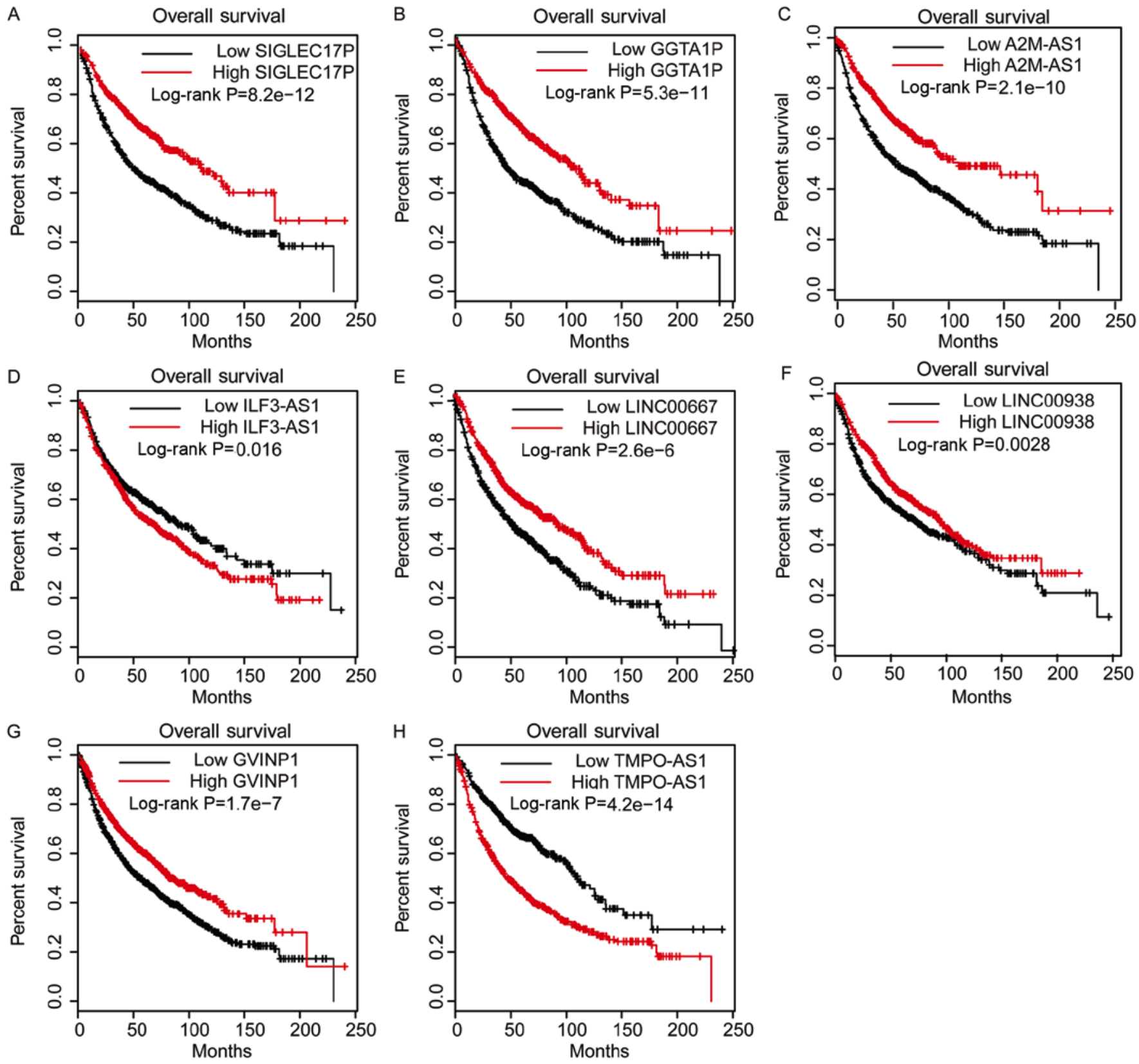

Figure 5. Dysregulation of DElncs is associated with survival time in NSCLC, as determined by analysis of the Kaplan-Meier-plotter database. Higher expression levels of (A) 1ncRNA-SIGLEC17P, (B) GGTA1P, (C) A2M-AS1, (F) LINC00938, and (G) GVINP1, and lower expression of (D) ILF3-AS1, (E) LINC00667 and (H) TMPO-AS1 were associated with longer survival times in patients with NSCLC. Differentially expressed lncRNAs; NSCLC, non-small cell lung cancer; lncRNA, long non-coding RNA.

reported to be downregulated, and to predict poor prognosis in prostate cancer. Additionally, lncRNA-CASC15 promoted the progression of various types of cancer, including melanoma, colon cancer, gastric cancer and hepatocellular carcinoma. TUG1 is widely reported to be an oncogene, regulating cancer cell proliferation, apoptosis, invasion and glutamine metabolism. In NSCLC, IncRNA-TUG1 was reported to be involved in regulating the growth and chemoresistance of NSCLC cells, in association with LIMK2b, BAX and HOXB7 (37). Of note, the majority of these lncRNAs, including EPB41L4A-AS2, ILF3-AS1, LINC00938, LINC00667 and A2M-AS1, have not been reported in human cancers.

In the present study, the association of these abnormally expressed lncRNAs with patient stage and survival time was assessed. It was revealed that the expression of IncRNA-GVINP1, RPL32P3, EPB41L4A-AS2, ILF3-AS1, LINC00938, LINC00667, A2M-AS1, GUSBP11, GGTA1P, BCRP3 and SIGLEC17P was suppressed, and that lncRNA-TMPO-AS1 expression was upregulated in high grade (stage III and IV) compared with low grade (stage II) NSCLC samples. Furthermore, Kaplan-Meier analysis illustrated that the overexpression of GVINP1, A2M-AS1, GGTA1P, SIGLEC17P, ILF3-AS1 and LINC00938, and the downregulation of TMPO-AS1 were associated with longer survival times in patients with LUAD. This demonstrated that these lncRNAs may serve as novel biomarkers for NSCLC.

For the most part, the functions of lncRNAs in cancer remain uninvestigated. Co-expression and bioinformatics 


\section{A A2M-AS1}

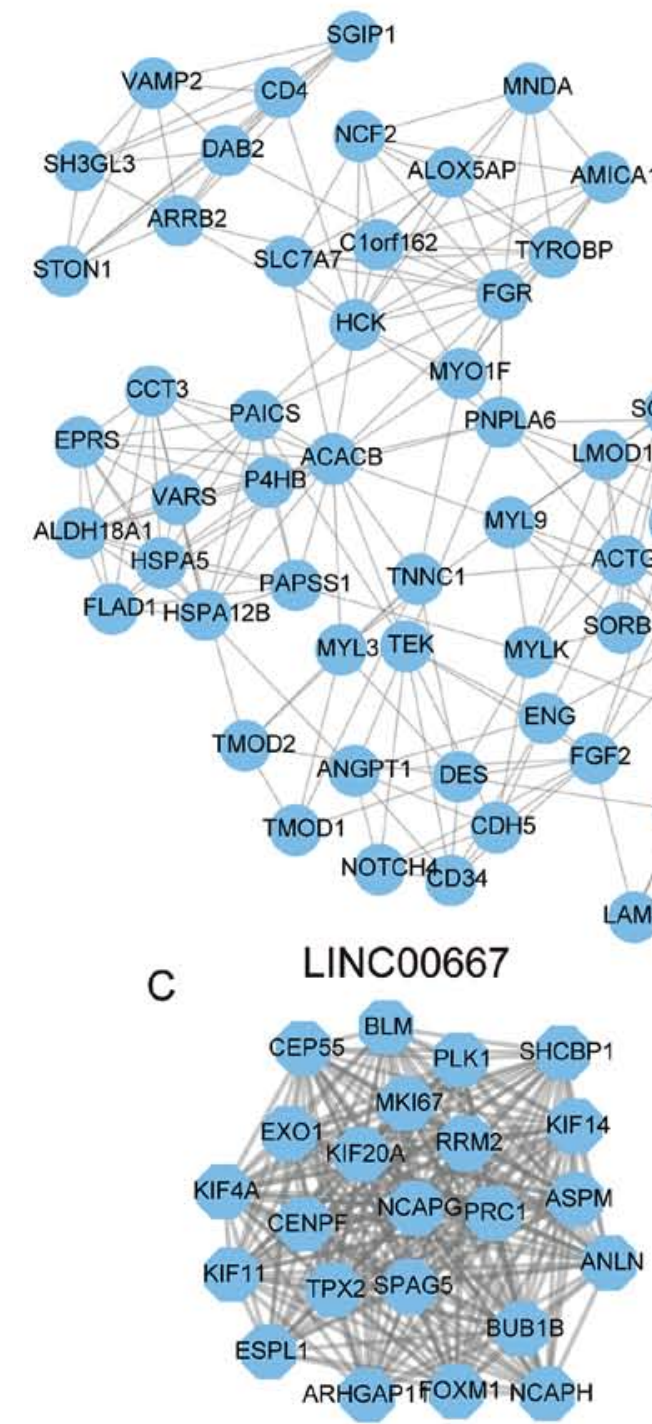

B

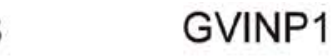

\section{TMPO-AS1}

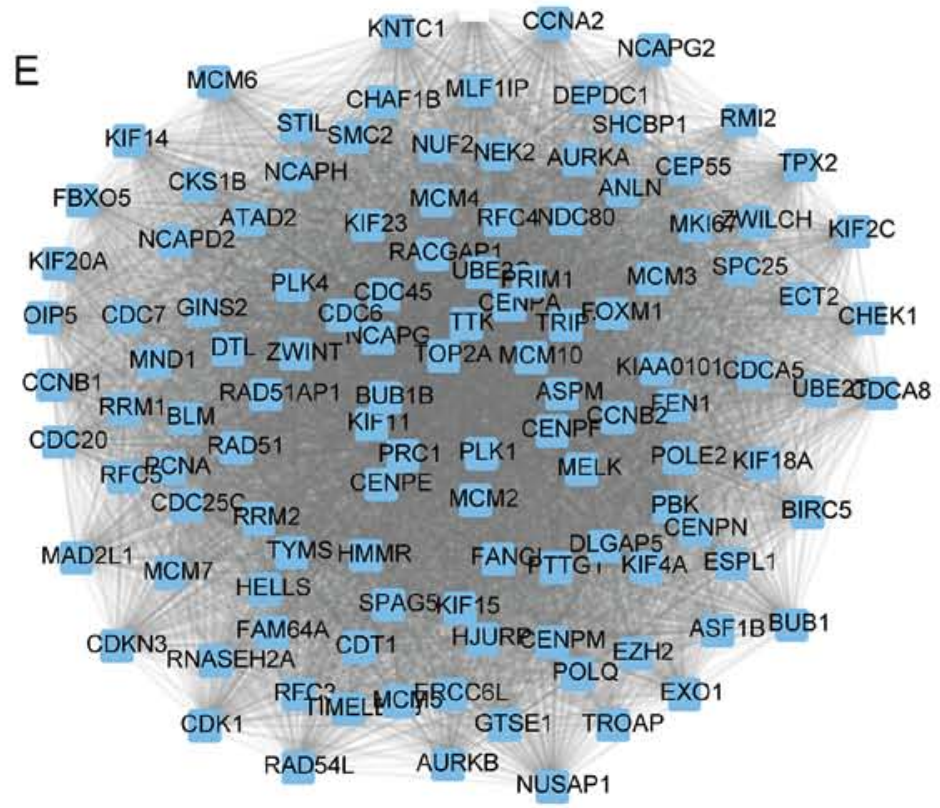

F

\section{GGTA1P} RRAS
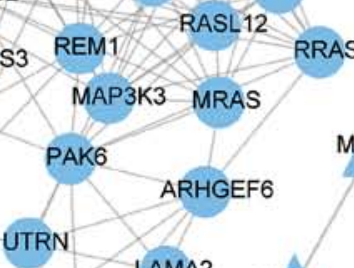

ARHGEF6
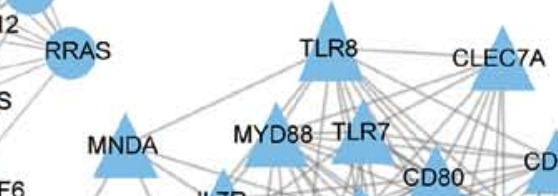

L7R CD80 CD83

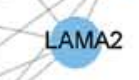

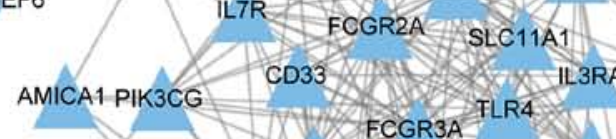

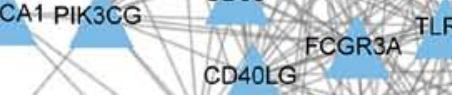

B 2 LAMC3
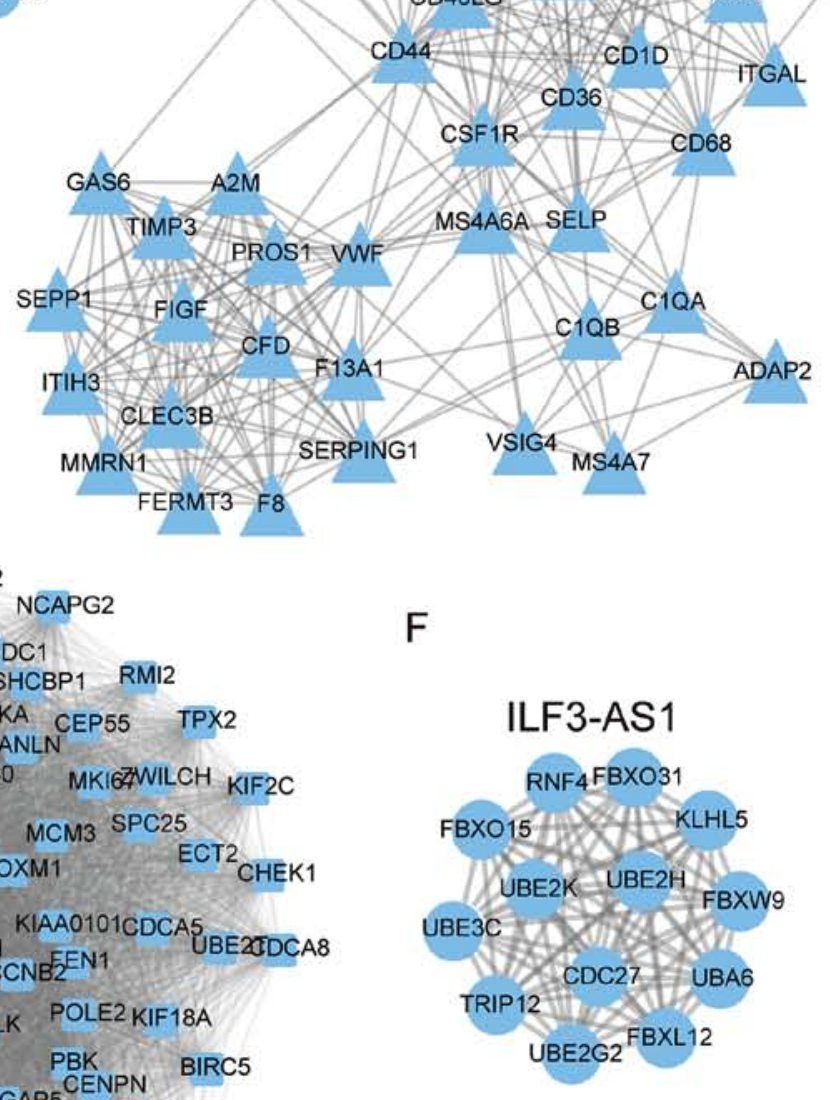

Figure 6. Construction of key lncRNA-associated PPI networks in NSCLC. lncRNA-associated PPI networks for (A) lncRNA-A2M-AS1, (B) GVINP1, (C) LINC00667, (D) GGTA1P, (E) TMPO-AS1 and (F) ILF3-AS1 in NSCLC. IncRNA, long non-coding RNA; PPI, protein-protein interaction; NSCLC, non-small cell lung cancer. 
A
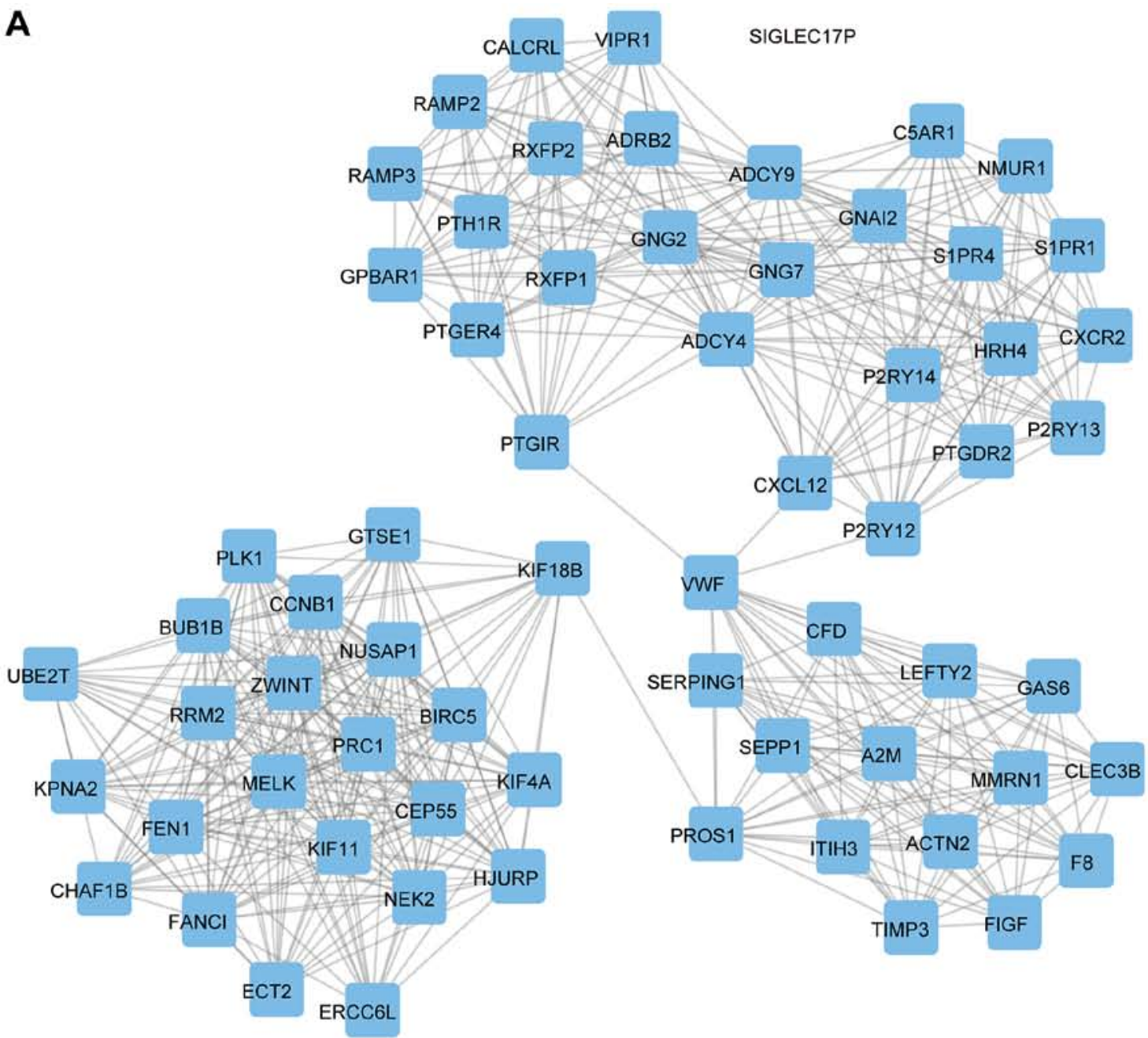

B
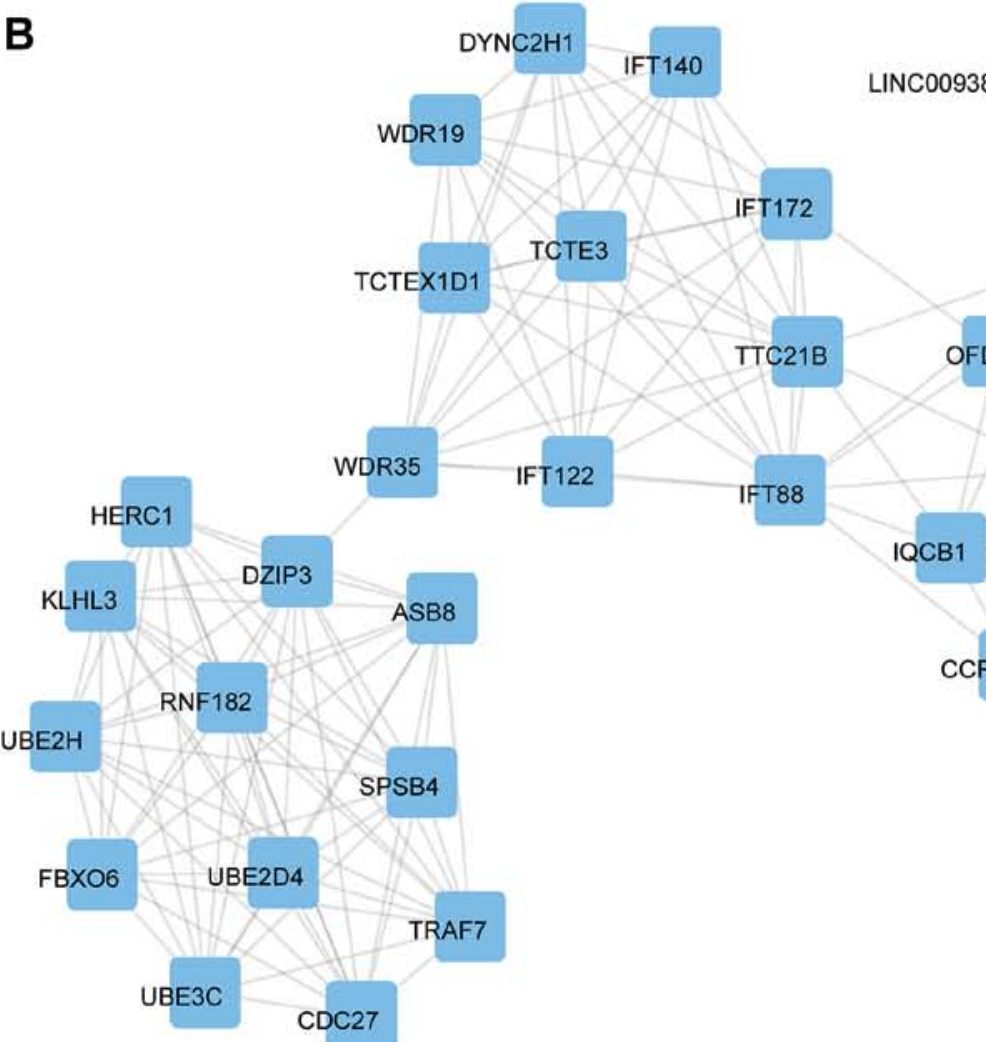

Figure 7. Construction of key lncRNA-associated PPI networks in NSCLC. IncRNA-associated PPI networks for (A) lncRNA-SIGLEC17P and (B) LINC00938 in NSCLC. lncRNA, long non-coding RNA; PPI, protein-protein interaction; NSCLC, non-small cell lung cancer. 
A

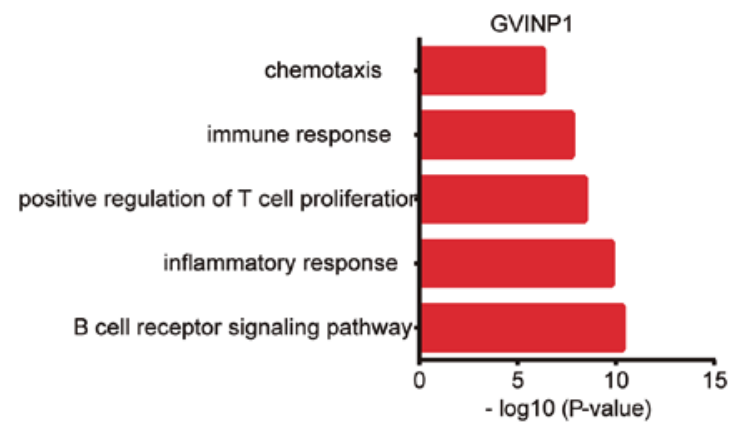

C

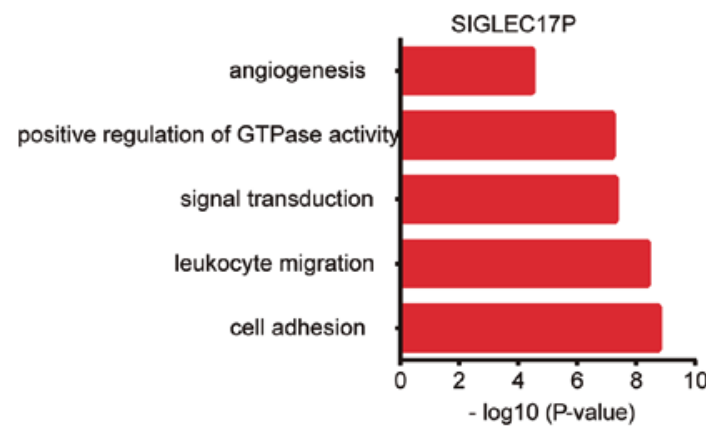

E

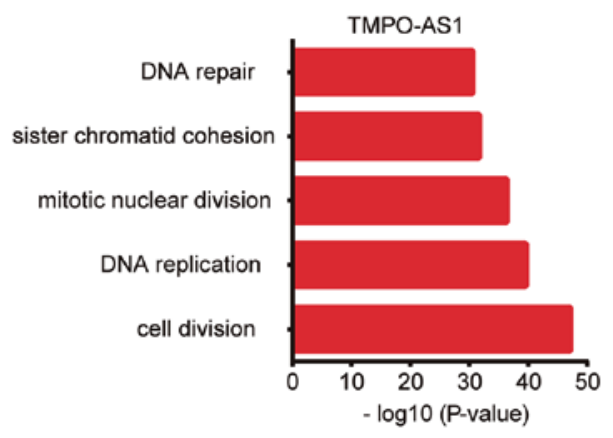

G

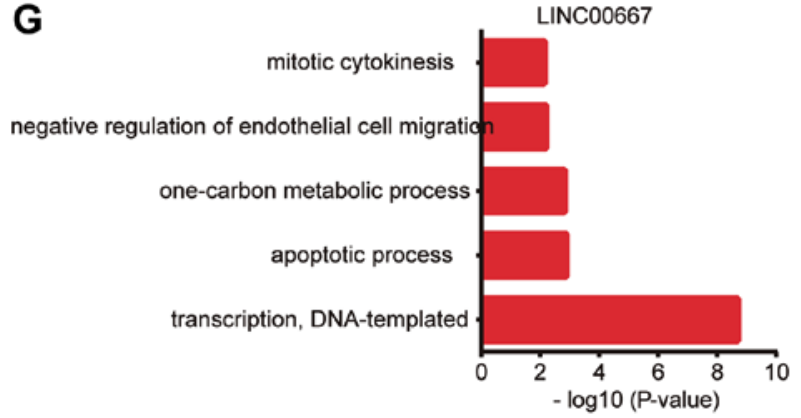

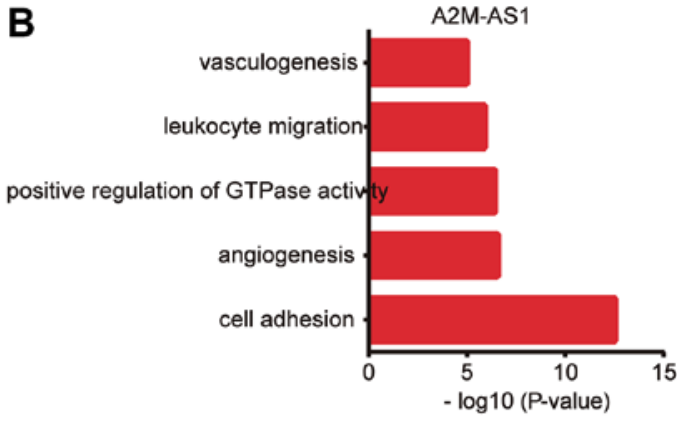

D

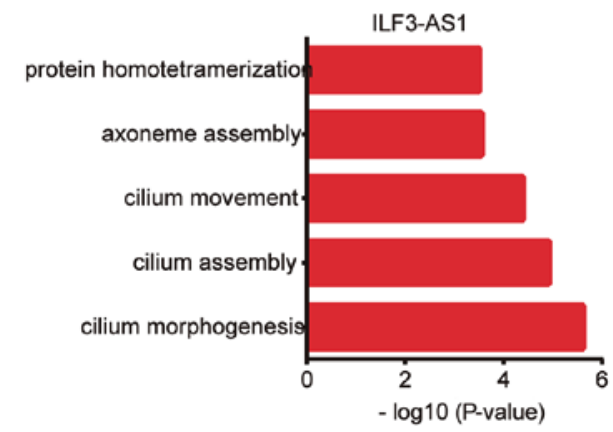

$\mathbf{F}$

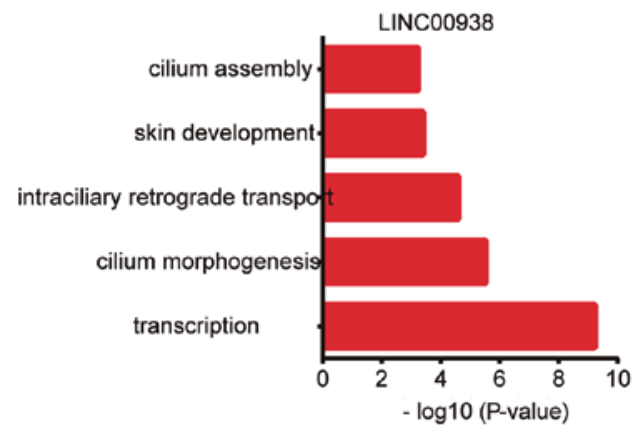

H

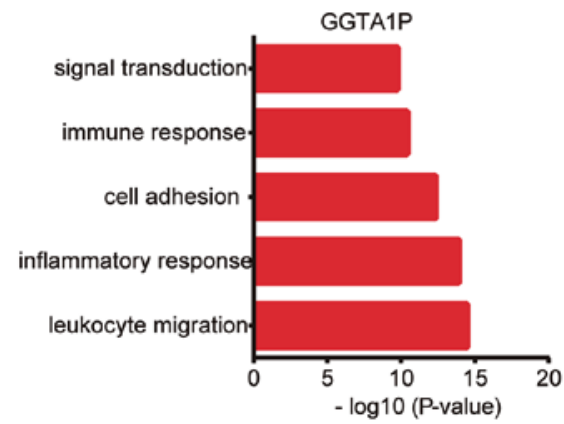

Figure 8. Bioinformatics analysis of the functional roles of key lncRNAs in NSCLC. Bioinformatics analysis revealed the functional roles of (A) IncRNA-GVINP1, (B) A2M-AS1, (C) SIGLEC17P, (D) ILF3-AS1, (E) TMPO-AS1, (F) LINC00938, (G) LINC00667 and (H) GGTA1P in NSCLC. NSCLC, non-small cell lung cancer; lncRNA, long non-coding RNA.

analysis has been widely used to identify the potential functions and molecular mechanisms of novel lncRNAs in human diseases $(38,39)$; using bioinformatics analysis, Jiao et al $(40)$ revealed the roles of IncRNAs associated with polycystic ovary syndrome. In the present study, IncRNA-associated PPI modules in NSCLC progression were constructed. GO bioinformatics analysis indicated that lncRNA-LINC00667 was involved in the regulation of transcription and apoptotic process, and that lncRNA-GVINP1 was involved in regulating $B$ cell receptor signaling and the inflammatory response. lncRNA-A2M-AS1 and SIGLEC17P were associated with cell adhesion regulation, and GGTA1P was associated with leukocyte migration, inflammatory response and immune response. IncRNA-ILF3-AS1 was involved in regulating cilium morphogenesis and cilium assembly, whilst LINC00938 was involved in regulating transcription and cilium morphogenesis, and TMPO-AS1, with cell cycle and DNA repair progression.

The present study identified potential, novel biomarkers for the progression of NSCLC. However, one limitation should be noted; the expression levels of these IncRNAs in NSCLC tumor and serum samples were not detected using clinical samples. Further validation of their expression in NSCLC samples is required, and may strengthen their prognostic value as potential biomarkers for NSCLC. 
In conclusion, 38 upregulated, and 31 downregulated NSCLC-associated IncRNAs were identified through comprehensive analysis of public datasets. A total of $12 \mathrm{lncRNAs}$ were associated with NSCLC progression. Furthermore, Kaplan-Meier-plotter analysis illustrated for the first time, that higher expression levels of IncRNA-GVINP1, A2M-AS1, GGTA1P, SIGLEC17P, ILF3-AS1 and LINC00938, and lower expression levels of lncRNA-TMPO-AS1 were associated with longer survival time in patients with NSCLC. Key lncRNA-associated PPI networks were constructed, and bioinformatics analysis performed to provide insights for the identification of novel biomarkers for NSCLC.

\section{Acknowledgements}

The authors would like to thank Dr Xuechao Wan (School of Life Science, Fudan University, China) for excellent technical and graphic design expertise.

\section{Funding}

The present study was supported by the National Natural Science Foundation of China (grant no. 81700092), the National Natural Science Foundation of China (grant no. 81700049), the China Postdoctoral Science Foundation (grant no. 2016M601603), the Shanghai Science and Technology Innovation Fund (grant no. 18140903802), the Shanghai Municipal Health and Family Planning Commission Research Fund (grant no. 20174Y0234), the Shanghai Municipal Health and Family Planning Commission Research Fund (grant no. 20174Y0094) and the Huashan Hospital affiliated to Fudan University Research Start-up Fund (grant no. 2016QD087).

\section{Availability of data and materials}

The datasets used and/or analyzed during the current study are available from the corresponding author on reasonable request.

\section{Authors' contributions}

WF and HZ conceived and designed the study. GS acquired the data, and TL analyzed the data. WF and WZ drafted the manuscript and were involved in the conception of the study. LL performed the statistical analysis. All authors read and approved the manuscript and agree to be accountable for all aspects of the research, ensuring that the accuracy and integrity of any part of the work was appropriately investigated and resolved.

\section{Ethics approval and consent to participate}

Not applicable.

\section{Patient consent for publication}

Not applicable.

\section{Competing interests}

The authors declare that they have no competing interests.

\section{References}

1. Lu Y, Zhao X, Liu Q, Li C, Graves-Deal R, Cao Z, Singh B, Franklin JL, Wang J, Hu H, et al: lncRNA MIR100HG-derived miR-100 and miR-125b mediate cetuximab resistance via Wnt/ $\beta$-catenin signaling. Nat Med 23: 1331-1341, 2017.

2. Zhang M, Weng W, Zhang Q, Wu Y, Ni S, Tan C, Xu M, Sun H, Liu C, Wei P and Du X: The lncRNA NEAT1 activates Wnt $/ \beta$-catenin signaling and promotes colorectal cancer progression via interacting with DDX5. J Hematol Oncol 11: 113, 2018.

3. Giraldez MD, Spengler RM, Etheridge A, Godoy PM, Barczak AJ Srinivasan S, De Hoff PL, Tanriverdi K, Courtright A, Lu S, et al: Comprehensive multi-center assessment of small RNA-seq methods for quantitative miRNA profiling. Nat Biotechnol 36: 746-757, 2018.

4. Beermann J, Piccoli MT, Viereck J and Thum T: Non-coding RNAs in development and disease: Background, mechanisms, and therapeutic approaches. Physiol Rev 96: 1297-1325, 2016

5. Zhang Y, Su X, Kong Z, Fu F, Zhang P, Wang D, Wu H, Wan X and Li Y: An androgen reduced transcript of IncRNA GAS5 promoted prostate cancer proliferation. PLoS One 12: e0182305, 2017.

6. Luo L, Tang H, Ling L, Li N, Jia X, Zhang Z, Wang X, Shi L, Yin J, Qiu N, et al: LINC01638 lncRNA activates MTDH-Twist1 signaling by preventing SPOP-mediated c-Myc degradation in triple-negative breast cancer. Oncogene 37: 6166-6167, 2018.

7. Yuan JH, Liu XN, Wang TT, Pan W, Tao QF, Zhou WP, Wang F and Sun SH: The MBNL3 splicing factor promotes hepatocellular carcinoma by increasing PXN expression through the alternative splicing of IncRNA-PXN-AS1. Nat Cell Biol 19: 820-832, 2017.

8. Zhou W, Chen X, Hu Q, Chen X, Chen Y and Huang L: Galectin-3 activates TLR4/NF- $\mathrm{KB}$ signaling to promote lung adenocarcinoma cell proliferation through activating lncRNA-NEAT1 expression. BMC Cancer 18: 580, 2018.

9. Schmitt AM and Chang HY: Long noncoding RNAs in cancer pathways. Cancer Cell 29: 452-463, 2016.

10. Xia Y, Yan Z, Wan Y, Wei S, Bi Y, Zhao J, Liu J, Liao DJ and Huang H: Knockdown of long noncoding RNA GHET1 inhibits cellcycle progression and invasion of gastric cancer cells. Mol Med Rep 18: 3375-3381, 2018.

11. Wu HF, Ren LG, Xiao JQ, Zhang Y, Mao XW and Zhou LF: Long non-coding RNA LINP1 promotes the malignant progression of prostate cancer by regulating p53. Eur Rev Med Pharmacol Sci 22: 4467-4476, 2018.

12. Wang P, Wu T, Zhou H, Jin Q, He G, Yu H, Xuan L, Wang X, Tian L, Sun Y, et al: Long noncoding RNA NEAT1 promotes laryngeal squamous cell cancer through regulating miR-107/CDK6 pathway. J Exp Clin Cancer Res 35: 22, 2016.

13. Zou SM, Li WH, Wang WM, Li WB, Shi SS, Ying JM and Lyu N: The gene mutational discrepancies between primary and paired metastatic colorectal carcinoma detected by next-generation sequencing. J Cancer Res Clin Oncol 144: 2149-2159, 2018

14. Cheng $\mathrm{K}$, Zhao Z, Wang G, Wang J and Zhu W: lncRNA GAS5 inhibits colorectal cancer cell proliferation via the miR1825p/FOXO3a axis. Oncol Rep 41: 2371-2380, 2018.

15. Huang Z, Lei W, Tan J and Hu HB: Long noncoding RNA LINC00961 inhibits cell proliferation and induces cell apoptosis in human non-small cell lung cancer. J Cell Biochem 119: 9072-9080, 2018

16. Gandhi L, Rodriguez-Abreu D, Gadgeel S, Esteban E, Felip E, De Angelis F, Domine M, Clingan P, Hochmair MJ, Powell SF, et al: Pembrolizumab plus chemotherapy in metastatic non-small-cell lung cancer. N Engl J Med 378: 2078-2092, 2018.

17. Miller VA, Hirsh V, Cadranel J, Chen YM, Park K, Kim SW, Zhou C, Su WC, Wang M, Sun Y, et al: Afatinib versus placebo for patients with advanced, metastatic non-small-cell lung cancer after failure of erlotinib, gefitinib, or both, and one or two lines of chemotherapy (LUX-Lung 1): A phase $2 \mathrm{~b} / 3$ randomised trial. Lancet Oncol 13: 528-538, 2012.

18. Lu Z, Li Y, Che Y, Huang J, Sun S, Mao S, Lei Y, Li N, Sun N and He J: The The TGF $\beta$-induced IncRNA TBILA promotes non-small cell lung cancer progression in vitro and in vivo via cis-regulating HGAL and activating S100A7/JAB1 signaling. Cancer Lett 432: 156-168, 2018.

19. Zhang X, Sun S, Pu JK, Tsang AC, Lee D, Man VO, Lui WM, Wong ST and Leung GK: Long non-coding RNA expression profiles predict clinical phenotypes in glioma. Neurobiol Dis 48: $1-8,2012$ 
20. Lu TP, Tsai MH, Lee JM, Hsu CP, Chen PC, Lin CW, Shih JY, Yang PC, Hsiao CK, Lai LC and Chuang EY: Identification of a novel biomarker, SEMA5A, for non-small cell lung carcinoma in nonsmoking women. Cancer Epidemiol Biomarkers Prev 19: 2590-2597, 2010.

21. Wei TY, Hsia JY, Chiu SC, Su LJ, Juan CC, Lee YC, Chen JM, Chou HY, Huang JY, Huang HM and Yu CT: Methylosome protein 50 promotes androgen- and estrogen-independent tumorigenesis. Cell Signal 26: 2940-2950, 2014

22. Sanchez-Palencia A, Gomez-Morales M, Gomez-Capilla JA, Pedraza V, Boyero L, Rosell R and Fárez-Vidal ME: Gene expression profiling reveals novel biomarkers in nonsmall cell lung cancer. Int J Cancer 129: 355-364, 2011.

23. Hou J, Aerts J, den Hamer B, van Ijcken W, den Bakker M, Riegman P, van der Leest C, van der Spek P, Foekens JA, Hoogsteden HC, et al: Gene expression-based classification of non-small cell lung carcinomas and survival prediction. PLoS One 5: e10312, 2010.

24. Huang da W, Sherman BT and Lempicki RA: Bioinformatics enrichment tools: Paths toward the comprehensive functional analysis of large gene lists. Nucleic Acids Res 37: 1-13, 2009.

25. Wu J, Weng Y, He F, Liang D and Cai L: IncRNA MALAT-1 competitively regulates miR-124 to promote EMT and development of non-small-cell lung cancer. Anticancer Drugs 29: 628-636, 2018

26. Qiu M, Xu Y, Yang X, Wang J, Hu J, Xu L and Yin R: CCAT2 is a lung adenocarcinoma-specific long non-coding RNA and promotes invasion of non-small cell lung cancer. Tumour Biol 35 $5375-5380,2014$

27. Yang L, Xie N, Huang J, Huang H, Xu S, Wang Z and Cai J: SIK1-LNC represses the proliferative, migrative, and invasive abilities of lung cancer cells. Onco Targets Ther 11: 4197-4206, 2018.

28. Zhao JM, Cheng W, He XG, Liu YL, Wang FF and Gao YF: Long non-coding RNA PICART1 suppresses proliferation and promotes apoptosis in lung cancer cells by inhibiting JAK2/STAT3 signaling. Neoplasma 65: 779-789, 2018.

29. Liu XH, Liu ZL, Sun M, Liu J, Wang ZX and De W: The long non-coding RNA HOTAIR indicates a poor prognosis and promotes metastasis in non-small cell lung cancer. BMC Cancer 13: 464, 2013.

30. Zhang Q, Li X, Li X, Li X and Chen Z: 1ncRNA H19 promotes epithelial-mesenchymal transition (EMT) by targeting miR-484 in human lung cancer cells. J Cell Biochem 119: 4447-4457, 2018.

31. Zhang Z, Liu T, Wang K, Qu X, Pang Z, Liu S, Liu Q and Du J: Down-regulation of long non-coding RNA MEG3 indicates an unfavorable prognosis in non-small cell lung cancer: Evidence from the GEO database. Gene 630: 49-58, 2017.
32. Lin PC, Huang HD, Chang CC, Chang YS, Yen JC, Lee CC, Chang WH, Liu TC and Chang JG: Long noncoding RNA TUG1 is downregulated in non-small cell lung cancer and can regulate CELF1 on binding to PRC2. BMC Cancer 16: 583, 2016.

33. Zhang Y, Zhang P, Wan X, Su X, Kong Z, Zhai Q, Xiang X, $\mathrm{Li} \mathrm{L}$ and $\mathrm{Li}$ Y: Downregulation of long non-coding RNA HCG11 predicts a poor prognosis in prostate cancer. Biomed Pharmacother 83: 936-941, 2016.

34. Yao XM, Tang JH, Zhu H and Jing Y: High expression of lncRNA CASC15 is a risk factor for gastric cancer prognosis and promote the proliferation of gastric cancer. Eur Rev Med Pharmacol Sci 21: 5661-5667, 2017

35. Chen CQ, Chen CS, Chen JJ, Zhou LP, Xu HL, Jin WW, Wu JB and Gao SM: Histone deacetylases inhibitor trichostatin A increases the expression of Dleu2/miR-15a/16-1 via HDAC3 in non-small cell lung cancer. Mol Cell Biochem 383: 137-148, 2013.

36. Shi H, Shi J, Zhang Y, Guan C, Zhu J, Wang F, Xu M, Ju Q, Fang $S$ and Jiang M: Long non-coding RNA DANCR promotes cell proliferation, migration, invasion and resistance to apoptosis in esophageal cancer. J Thorac Dis 10: 2573-2582, 2018.

37. Niu Y, Ma F, Huang W, Fang S, Li M, Wei T and Guo L: Long non-coding RNA TUG1 is involved in cell growth and chemoresistance of small cell lung cancer by regulating LIMK2b via EZH2. Mol Cancer 16: 5, 2017.

38. Chen W, Zhang X, Li J, Huang S, Xiang S, Hu X and Liu C: Comprehensive analysis of coding-lncRNA gene co-expression network uncovers conserved functional lncRNAs in zebrafish. BMC Genomics 19 (Suppl 2): S112, 2018.

39. Fang L, Wang $\mathrm{H}$ and Li P: Systematic analysis reveals a IncRNA-mRNA co-expression network associated with platinum resistance in high-grade serous ovarian cancer. Invest New Drugs 36: 187-194, 2018.

40. Jiao J, Shi B, Wang T, Fang Y, Cao T, Zhou Y, Wang X and Li D: Characterization of long non-coding RNA and messenger RNA profiles in follicular fluid from mature and immature ovarian follicles of healthy women and women with polycystic ovary syndrome. Hum Reprod 33: 1735-1748, 2018.

(7) $\odot$ This work is licensed under a Creative Commons Attribution-NonCommercial-NoDerivatives 4.0 International (CC BY-NC-ND 4.0) License. 